\title{
Coordinating Solvent-Assisted Synthesis of Phase-Stable Perovskite Nanocrystals with High Yield Production for Optoelectronic Applications
}

\author{
Min Kim,* Daniele Cortecchia, Tetiana Borzda, Wojciech Mróz, Luca Leoncino, David Dellasega, \\ Soo-Hyoung Lee, and Annamaria Petrozza*
}

Cite This: Chem. Mater. 2021, 33, 547-553

Read Online

ACCESS | Lلll Metrics \& More | 回 Article Recommendations | st Supporting Information

ABSTRACT: Inorganic perovskite nanocrystals (NCs) have shown good potential as an emerging semiconducting building block owing to their excellent optoelectronic properties. However, despite extensive studies on their structure-dependent optical properties, they still suffer severely from chemical and phase instabilities in ambient conditions. Here, we report a facile method for the synthesis of mixed halide inorganic perovskite NCs based on recrystallization in an antisolvent mixture in an ambient atmosphere, at room temperature. We introduced an alcohol-derivative solvent, as a secondary antisolvent in the solvent mixture, which crystallizes

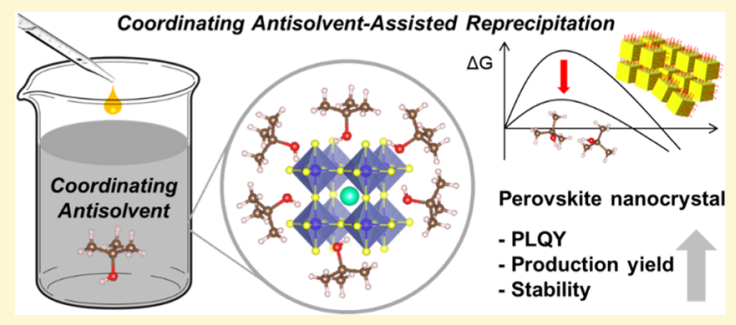
at room temperature. This mediates and facilitates the perovskite crystallization, leading to a high chemical yield and stability. We demonstrate that this secondary antisolvent establishes intermolecular interactions with lead halide salt, which successfully stabilizes the $\gamma$-dark phase of perovskite by encapsulating NCs in a solution and thin film. This allows us to produce concentrated NC solutions with a photoluminescence quantum yield of $70 \%$. Finally, we fabricate $\mathrm{CsPb}_{2} \mathrm{Br} \mathrm{NCs}$ (optical bandgap $1.88 \mathrm{eV}$ ) solar cells, which showed a stabilized photovoltaic performance in ambient conditions, without encapsulation, showing a $V_{\text {oc }}$ of $1.32 \mathrm{~V}$.

\section{INTRODUCTION}

Metal halide perovskite nanocrystals (NCs) have gathered immense attention as materials with highly tunable chemistry and unique optoelectronic properties such as the so-called defect tolerance. ${ }^{1-3}$ They have been implemented in a variety of optoelectronic applications, like light-emitting diodes, solar cells, white phosphors, and solar concentrators. ${ }^{4-8}$ Among them, the Cs-based all-inorganic lead halide perovskites, $\mathrm{CsPbX}_{3}(\mathrm{X}=\mathrm{I}, \mathrm{Br}, \mathrm{Cl})$, without a volatile organic component, has been the result of particular interest due to their high potential in terms of thermal stability. ${ }^{9-11}$ However, they suffer from severe chemical and phase instability, especially when targeting narrower band gap semiconductors because iodidecontaining inorganic perovskites could be easily degraded to the nonphotoactive $\delta$-phase under illumination in ambient conditions. $^{12,13}$

The most widely used synthesis method for mixed halide inorganic perovskite NC has been hot-injection (HI) synthesis. $^{1-3,14}$ The HI holds all of the control over the reaction environment such as temperature, vacuum, and inert gas filling, so it is effective to stabilize the desirable perovskite phase of $\mathrm{CsPbX}_{3}$ perovskite NCs. ${ }^{15}$ However, at the same time, the $\mathrm{HI}$ method presents limitations in terms of scalability and an absolute yield due to the need for vacuum and inert-conditions equipment, a limited solubility in an inert solvent, and the temperature inhomogeneity in the reaction vessel. ${ }^{16,17}$ Thus, a low-temperature synthetic method compatible with ambient conditions would be ideal to improve the productivity of the NC synthesis. For a suitable replacement, the ligand-assisted reprecipitation (LARP) method has been employed to synthesize various types of perovskite NCs. ${ }^{18,19}$ The LARP procedure simply includes dropping a low amount of perovskite precursors directly into the antisolvent, which can crystallize perovskites during a strong agitation. In particular, this LARP method can be carried out in ambient conditions and at room temperature (RT) and showed high-performance optoelectronic properties of the perovskite semiconductors. ${ }^{20,21}$ However, LARP also has a low reaction yield because it is based on the principle of solubility difference between the antisolvent and perovskite precursor solution. So far, this has hampered the use of such methodology for the production of materials and their photovoltaic applications.

Here, we present a novel LARP method using an antisolvent mixture that has a secondary alcohol-based solvent in the benzene-based antisolvent. The secondary solvent is tert-butyl alcohol $(t-\mathrm{BuOH})$, which crystallizes at room temperature due

Received: August 26, 2020

Revised: December 19, 2020

Published: January 6, 2021 
(a)

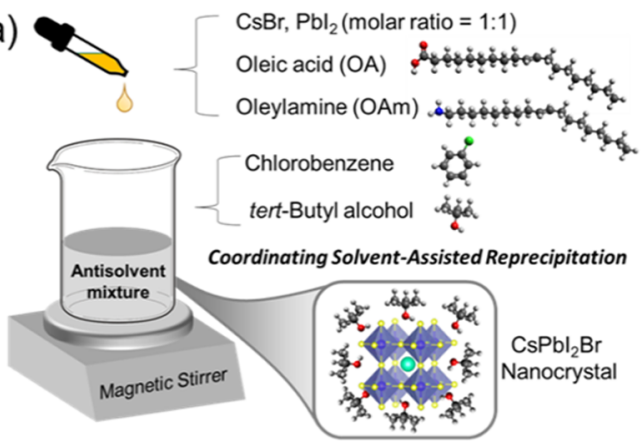

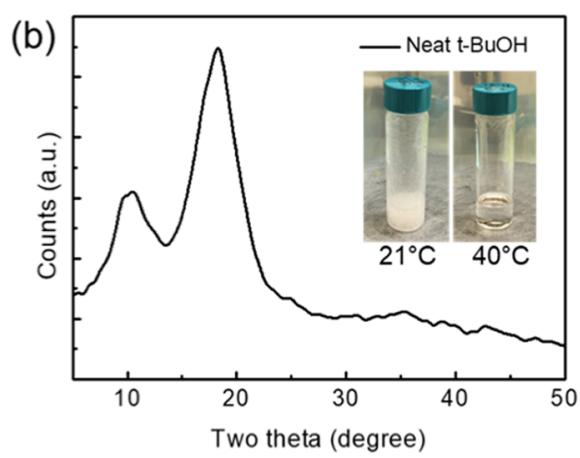

Figure 1. (a) Schematic representation of the perovskite nanocrystal synthesis based on ligand-assisted reprecipitation at room temperature in ambient conditions using mixed antisolvents with either toluene or chlorobenzene and tert-butyl alcohol $(t-\mathrm{BuOH})$. (b) X-ray diffraction $(\mathrm{XRD})$ of the neat $t-\mathrm{BuOH}$ droplet on a silicon wafer at room temperature. Inset: photographs of the vials containing the neat $t$ - $\mathrm{BuOH}$ solvent at temperatures of 21 and $40{ }^{\circ} \mathrm{C}$.

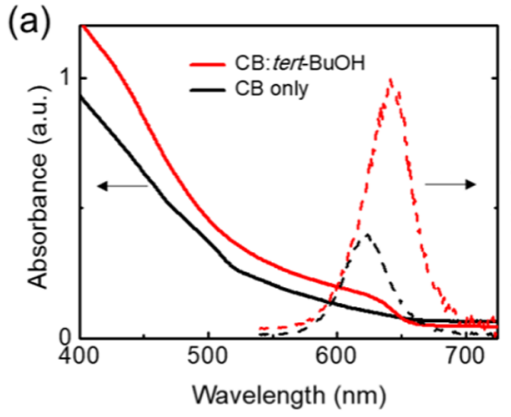

(c)

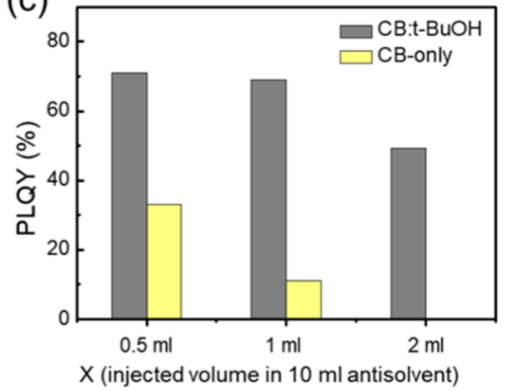

(b)

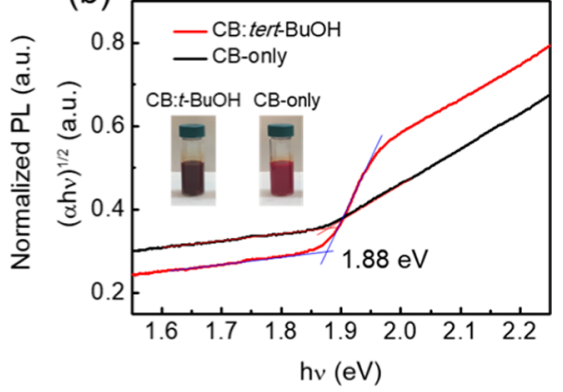

(d)

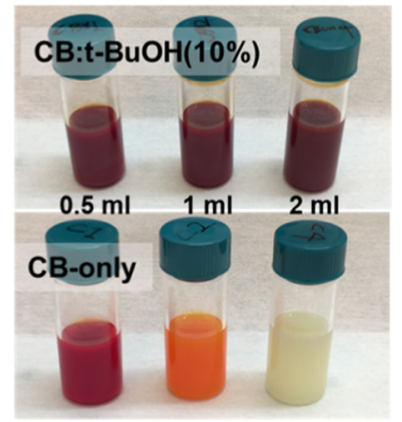

Figure 2. (a) Absorbance and PL spectra of $\mathrm{CsPbI}_{2} \mathrm{Br}$ NCs prepared from CB-only and $\mathrm{CB} / t$ - $\mathrm{BuOH}$. (b) Tauc's plots of the CsPbI $\mathrm{Br}^{\mathrm{N} C}$ solutions prepared from $\mathrm{CB}$-only or $\mathrm{CB} / \mathrm{t}-\mathrm{BuOH}$. Insets are photographs of the $\mathrm{CsPb}_{2} \mathrm{Br} \mathrm{NC}$ solutions. (c) Photoluminescence quantum yield (PLQY) as a function of the injection volume of perovskite solution into the antisolvent. (d) Photographs of the $\mathrm{CsPb}_{2} \mathrm{Br} \mathrm{NC}$ solutions at different ratios of the injected precursor volume to antisolvent volume.

to its high melting point above the room temperature. During the injection of the perovskite precursor into the mixed antisolvent, the $t-\mathrm{BuOH}$ solvent acts as a crystallization nucleus and facilitates the crystallization in the perovskite black $\gamma$ phase. We find that $t-\mathrm{BuOH}$ also stabilizes the perovskite NCs by passivating defect sites on the surface. Eventually, we fabricated $\mathrm{CsPbI}_{2} \mathrm{Br} \mathrm{NC}$ solar cells, which showed improved open-circuit voltage and good air stability compared to polycrystalline film device without encapsulation and to perovskite NC solar cells reported. ${ }^{22,23}$

\section{RESULTS AND DISCUSSION}

$\mathrm{CsPb}_{2} \mathrm{Br} \mathrm{NCs}$ are synthesized through a modified LARP protocol at room temperature with a relative humidity of 40$60 \%$. The perovskite precursor solution containing $\mathrm{CsBr}, \mathrm{PbI}_{2}$ (molar ratio $=1: 1)$, oleic acid $(\mathrm{OA})$, and oleylamine $(\mathrm{OAm})$ in dimethylformamide (DMF) was added to a vigorously stirred antisolvent mixture of chlorobenzene (CB) and $t-\mathrm{BuOH}(10 \%$ in volume; see Figure S2 and Table S1 for the optimization of the mixture ratio). The crystallization of the perovskite NCs is completed in a few seconds after the injection (Figure 1a). The secondary antisolvent, $t-\mathrm{BuOH}$, solidifies and crystallizes at room temperature (RT) due to its relatively high melting point at $26{ }^{\circ} \mathrm{C}$ (Figure $1 \mathrm{~b}$ ), thus forming small crystals in the solvent mixture. This feature has been exploited for isothermal crystallization in frozen aqueous $t-\mathrm{BuOH}$ solutions for the fabrication of freeze-dried drug products. ${ }^{24}$ Here, we utilize the formation of such $t$-BuOH crystallites to act as nuclei in the antisolvent and change the crystallization behavior to heterogeneous nucleation and growth of perovskite nanocrystals (Figure $\mathrm{S} 1$ ), resulting in a high yield of $\mathrm{CsPbI}_{2} \mathrm{Br} \mathrm{NC}$ formation. We obtained a chemical yield as high as $92 \%$ in the case of the $\mathrm{CB} / \mathrm{t}$ - $\mathrm{BuOH}$ solvent mixture, while the $\mathrm{CB}$-only solvent showed only a $71 \%$ yield. The chemical yield was determined by comparing the mass of the final reaction product with the expected weight for $0.1 \mathrm{mmol}$ of the product. 
(a)

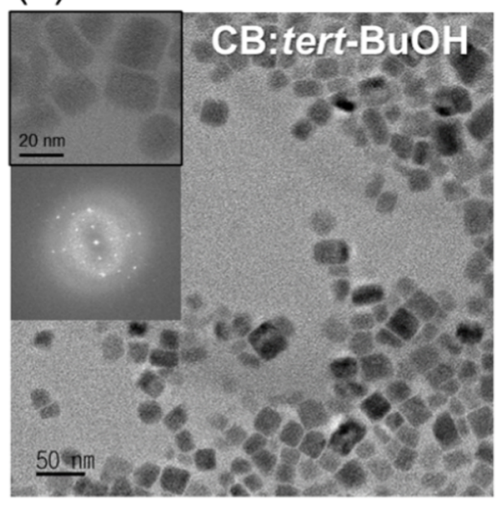

(b)

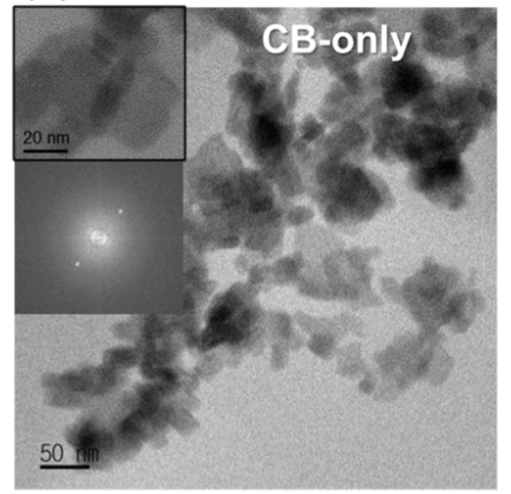

(c)

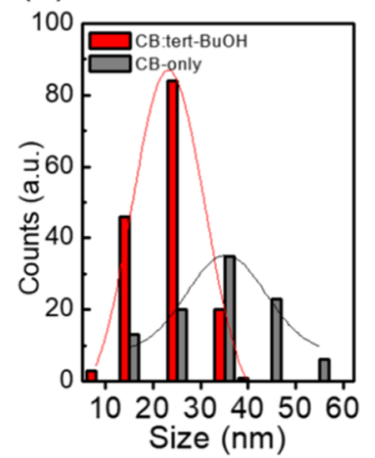

(d)

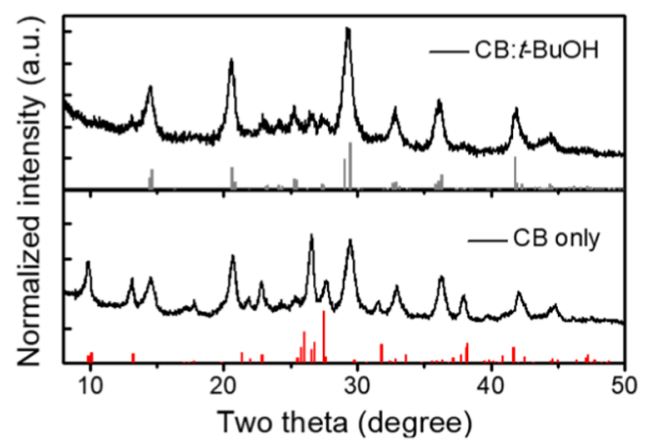

Figure 3. (a, b) BF-TEM images of the $\mathrm{CsPbI}_{2} \mathrm{Br}$ NCs deposited on carbon-coated grids. Insets are TEM images in higher magnification and their fast Fourier transform (FFT) patterns. (c) Statistics of the size of $\mathrm{CsPbI}_{2} \mathrm{Br}$ NCs. (d) XRD diffraction patterns of the $\mathrm{CsPbI}{ }_{2} \mathrm{Br} \mathrm{NC}$ films.

Figure 2a shows the ultraviolet-visible (UV-vis) absorbance and photoluminescence (PL) spectra of the samples obtained soon after the injection into the $\mathrm{CB}$-only or the $\mathrm{CB} / t$ $\mathrm{BuOH}$ mixture solvent. The use of $\mathrm{CB}$-only as antisolvent leads, as already reported in the literature, ${ }^{19,25}$ to a solution with a broad and weak absorption edge and an emission peaked at $622 \mathrm{~nm}$ (full width at half-maximum (FWHM) = $36.6 \mathrm{~nm}$ ). The absorbance of the $\mathrm{CsPbI}_{2} \mathrm{Br} \mathrm{NC}$ solution prepared from an optimized $\mathrm{CB} / t-\mathrm{BuOH}$ mixture (see Figure S2) shows a steeper optical absorption edge at $650 \mathrm{~nm}$ and a significant increase in the absorbance over the entire absorption range (Figure $2 \mathrm{~b}$ ). The $\mathrm{PL}$ spectrum is about 20 $\mathrm{nm}$ red-shifted $(639 \mathrm{~nm})$ with respect to the control CB-only sample, and importantly, it presents a photoluminescence quantum yield (PLQY) of $70 \%$, which doubles the PLQY of the CB-only NC solution. Bright-field transmission electron microscopy (BF-TEM) images suggest that both solutions are made of NC of about $30 \mathrm{~nm}$ size, which confirms that the optical features observed are due to higher purity of darkbrown perovskite phase in the presence of $t-\mathrm{BuOH}$ (Figure $3 a, b)$. This superior crystallization of inorganic perovskite in $\mathrm{CB} / \mathrm{t}-\mathrm{BuOH}$ is even more evident in the case of larger loading of the perovskite precursor solution in the antisolvents (Figure $2 \mathrm{c}, \mathrm{d})$. We could stably increase the chemical yield of the blackphase NC formation by simply increasing the injection volume of perovskite precursor solution up to $2 \mathrm{~mL}$ into $10 \mathrm{~mL}$ of the antisolvent mixture, which maintains high PLQYs until $2 \mathrm{~mL}$ of the volume injection in the $10 \mathrm{~mL}$ of $\mathrm{CB} / \mathrm{t}-\mathrm{BuOH}$ antisolvent. On the other hand, the CB-only NC turns orange and yellow with the injection of up to $2 \mathrm{~mL}$ of perovskite solution into $10 \mathrm{~mL}$ of $\mathrm{CB}$.
BF-TEM was used to determine the size and morphology of the $\mathrm{CsPbI}_{2} \mathrm{Br} \mathrm{NCs}$ (Figure 3a,b). Statistical analysis of these TEM images suggests that the CB-only NCs have a broad size distribution (average around $35.3 \mathrm{~nm}$ ), while the $\mathrm{CB} / \mathrm{t}-\mathrm{BuOH}$ NCs appear to have a narrower size distribution (average around $23.1 \mathrm{~nm}$ ) (Figure $3 \mathrm{c}$ ). Furthermore, the $\mathrm{CB} / \mathrm{t}-\mathrm{BuOH}$ NCs show the cubic morphology of individual NCs, having high crystallinity with the orthorhombic phase, as evidenced by the FFT image (Figure 3a inset). On the other hand, the CBonly NCs exhibit irregular round shape with a broad size distribution, which also shows a lower degree of crystallinity. The TEM images show that the CB-only NCs are more likely to aggregate compared to the $\mathrm{CB} / \mathrm{t}$ - $\mathrm{BuOH} \mathrm{NCs}$.

Figure $3 \mathrm{~d}$ shows the XRD pattern of the drop-cast sample obtained from $\mathrm{CB}$-only and $\mathrm{CB} / \mathrm{t}-\mathrm{BuOH}$. The $\mathrm{CB} / \mathrm{t}-\mathrm{BuOH}$ NC film exhibits a distorted lower-symmetry orthorhombic perovskite structure in which the Bragg peaks at 14.16, 14.33, 28.54 , and $28.89^{\circ}$ can be assigned to the (200), (110), (400), and (220) planes of the $\gamma-\mathrm{CsPbI}_{3}$ crystallites, respectively. ${ }^{26}$ This orthorhombic crystal structure of the NCs, Pbnm (no. 62 ), was confirmed by a reference (Figure S3). On the other hand, the diffraction profile of the CB-only NC displays a polymorph of a nonperovskite yellow $\delta$-phase (space group Pnma) and $\gamma$ - $\mathrm{CsPb}_{3}$ crystallites, which agrees with the absorption spectra.

To investigate the molecular interaction between $t-\mathrm{BuOH}$ and perovskite NCs, ${ }^{1} \mathrm{H}$ NMR spectra of the $t-\mathrm{BuOH}$ in toluene- $d_{8}$ solution were recorded with and without the $\mathrm{CsPbI}_{2} \mathrm{Br}$ NCs. Figure $4 \mathrm{a}$ presents the ${ }^{1} \mathrm{H}$ NMR spectra of $t$ $\mathrm{BuOH}$ in toluene- $\mathrm{d} 8$, where the peak at $1.41 \mathrm{ppm}$ is assigned to $-\mathrm{OH}$ and the peak at $1.12 \mathrm{ppm}$ is assigned to $-\mathrm{CH}_{3}$. When 

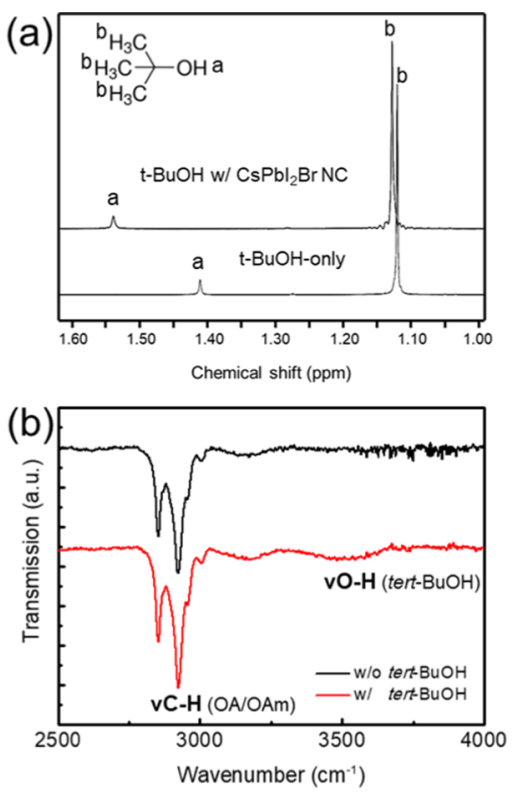

Figure 4. (a) Proton NMR spectra of $t-\mathrm{BuOH}$ in deuterated toluene with and without the $\mathrm{CsPbI}_{2} \mathrm{Br}$ NCs. (b) Transmission Fourier transform infrared (FT-IR) spectra of the $\mathrm{CsPbI}_{2} \mathrm{Br}$ NCs prepared on a silicon substrate.

$\mathrm{CsPb}_{2} \mathrm{Br}$ NCs were added into the solution, the $-\mathrm{OH}$ peak was downfield-shifted to $1.54 \mathrm{ppm}$. Such changes in chemical shifts indicate the formation of a hydrogen bond between $t$ $\mathrm{BuOH}$ and the perovskite $\mathrm{NC}$ surface. ${ }^{27}$

Compared to chlorobenzene, $t-\mathrm{BuOH}$ has an interaction with perovskite precursors, while acting as an antisolvent, due to its relatively high dielectric constant (Table 1). In other

Table 1. Properties of Solvents Used for the NC Synthesis ${ }^{28}$

\begin{tabular}{lrrcc}
\multicolumn{1}{c}{ solvent } & $\begin{array}{c}\mathrm{mp} \\
\left({ }^{\circ} \mathrm{C}\right)\end{array}$ & $\begin{array}{c}\mathrm{bp} \\
\left({ }^{\circ} \mathrm{C}\right)\end{array}$ & $\begin{array}{c}\text { dielectric } \\
\text { constant }\end{array}$ & $\begin{array}{c}\text { donor number } \\
(\mathrm{kcal} / \mathrm{mol})\end{array}$ \\
toluene & -95 & 110 & 2.38 & 0.1 \\
chlorobenzene & -45 & 131 & 5.62 & 3.3 \\
$\begin{array}{l}\text { tert-butyl } \\
\text { alcohol }\end{array}$ & 25 & 82 & 10.9 & 21.9 \\
DMF & -60 & 152 & 36.7 & 26.6 \\
\hline
\end{tabular}

words, we can compare the coordinating ability of the solvent with lead halide salts based on the donor number $\left(D_{N}\right)$, which describes the strength of interactions between Lewis base solvents and the soft Lewis acid $\mathrm{Pb}^{2+}$ in solution. ${ }^{28}$ We confirm that the $t-\mathrm{BuOH}$ solvent has a higher $D_{\mathrm{N}}$ than that of $\mathrm{CB}$ but lower than that of DMF, which means that $t-\mathrm{BuOH}$ weakly coordinates with $\mathrm{Pb}^{2+}$ and subsequently passivates the surface of perovskite NCs.

Fourier transform infrared (FT-IR) spectroscopy was also used to confirm the presence of $t-\mathrm{BuOH}$ after the deposition of $\mathrm{NCs}$ on the substrates (Figure $4 \mathrm{~b}$ ). $\mathrm{CsPbI}_{2} \mathrm{Br}$ capped with $\mathrm{OA}$ and OAm exhibited stretching vibration modes for methylene groups at 2923 and $2854 \mathrm{~cm}^{-1}$. On the other hand, the $\mathrm{CB} / t$ $\mathrm{BuOH} \mathrm{NC}$ film showed a clear peak at $\sim 3500 \mathrm{~cm}^{-1}$, corresponding to the $\mathrm{O}-\mathrm{H}$ stretching vibration mode of $t$ $\mathrm{BuOH}$, albeit at a lower intensity, which was confirmed by comparing it with the FT-IR spectra of $t$ - $\mathrm{BuOH}$ only (Figure S6). This indicates that $t-\mathrm{BuOH}$ exists as a ligand attached to the surface of perovskite NCs during the NC deposition.
Now, we discuss the stability of the perovskite phase of the $\mathrm{CsPbI}_{2} \mathrm{Br} \mathrm{NCs}$ prepared from $\mathrm{CB} / t-\mathrm{BuOH}$ in ambient conditions. The NC solutions dispersed in $\mathrm{CB}$ are stored at ambient conditions (air with RH 40-60\%, $T \sim 22{ }^{\circ} \mathrm{C}$ ) over 1 month. The inset in Figure 5a shows photographs of the $\mathrm{CsPbI}_{2} \mathrm{Br} \mathrm{NC}$ solutions in vials captured on day 1 and day 34 . During this period, the color of the CB-only NC solution changes from orange on day 1 to yellow color gradually, indicating a change in the optical band gap of the film with a continuous blue shift to $530 \mathrm{~nm}$ on day 17. The absorption edge at $530 \mathrm{~nm}$ is in agreement with the band edge of $\mathrm{CsPbBr}$. Therefore, this continuous peak shift suggests that the $\mathrm{CsPbI}_{2} \mathrm{Br}$ phase goes through halide segregation, and the iodide-rich phase collapses while only the bromide-rich phase becomes dominant because $\mathrm{CsPbBr}_{3}$ is a more thermodynamically stable phase than $\mathrm{CsPbI}_{3}{ }^{29}$

On the other hand, the $\mathrm{CB} / t-\mathrm{BuOH} \mathrm{CsPbI}_{2} \mathrm{Br}$ NCs showed significantly improved stability of the black phase when stored under the same ambient conditions. Figure $5 b$ shows the photographs of the NC solutions captured over 34 days, suggesting no color change. The optical absorption spectra monitored with time reveal improved stability, with an absorption edge maintained at $650 \mathrm{~nm}$. Importantly, the black phase remained stable also in the solid state. The XRD patterns of the $\mathrm{CB} / t-\mathrm{BuOH} \mathrm{CsPbI}{ }_{2} \mathrm{Br} \mathrm{NC}$ films were almost unchanged when stored for a week under the same ambient conditions, while the $\mathrm{CB}$-only $\mathrm{CsPbI}_{2} \mathrm{Br} \mathrm{NC}$ film shows a color change with the development of the nonperovskite $\delta$-phase diffraction peak (Figure 5c,d). Such an improvement in the stability of the black phase at room temperature is of significant importance for optoelectronic applications including solar cells.

We fabricated $\mathrm{CsPb}_{2} \mathrm{Br} \mathrm{NC}$ solar cells using a thin layer of $\mathrm{TiO}_{2}$ as the electron acceptor and poly(3-hexylthiophene) as the hole-transporting layer (HTM). ${ }^{30}$ The choice of the HTM was dictated by stability issues since we noticed a faster degradation of the NC films interfaced with Spiro-ometad, a topic that deserves per se further investigation (Figure 6a). The NC active layer was deposited by multiple spin coating with washing steps of dipping in $\mathrm{Pb}\left(\mathrm{NO}_{3}\right)_{2} /$ methyl acetate solvent to wash out surface ligands, reaching a thickness of around $200 \mathrm{~nm}$. The NC solar cell was tested under ambient conditions, delivering a power conversion efficiency (PCE) of $4.54 \%$ with an open-circuit voltage $\left(V_{\mathrm{oc}}\right)$ of $1.35 \mathrm{~V}$ (Figure $6 \mathrm{~b}$ ), which showed a small energy loss $\left(E_{\text {loss }}=E_{\mathrm{g}}-q V_{\text {oc }}\right.$ where $E_{\mathrm{g}}$ is the band gap and $q$ is the electron charge) of $0.53 \mathrm{eV}$ compared to the other reported $\mathrm{CsPbI}_{2} \mathrm{Br} \mathrm{NC}$ devices. ${ }^{22,23,31}$ Clearly, given the low fill factor and photocurrent of $56 \%$, and $6.0 \mathrm{~mA} / \mathrm{cm}^{2}$, respectively, the NC device process is still not fully optimized. We compared the stability of the NC device with that of a solar cell made of the $\mathrm{CsPbI}_{2} \mathrm{Br}$ compact film processed from the $\mathrm{CsBr} / \mathrm{PbI}_{2}$ solution in dimethyl sulfoxide (DMSO) with thermal treatment at $250{ }^{\circ} \mathrm{C}$. They showed a stark contrast. The compact film device degraded quickly even during the measurement, forming a yellow phase under the exposure to illumination (Figures $6 \mathrm{c}$ and S9). ${ }^{32-34}$ Importantly, to the best of our knowledge, solar cells made of perovskite $\mathrm{NC}$ are tested mainly in an inert environment due to the accelerated degradation. ${ }^{6,12,13,23,35} \mathrm{We}$ also measured the electroluminescence in ambient conditions (Figure 6d). The EL showed a $\mathrm{CsPbI}_{2} \mathrm{Br}$ spectrum in agreement with the photoluminescence spectrum. These spectra provide direct 
(a)
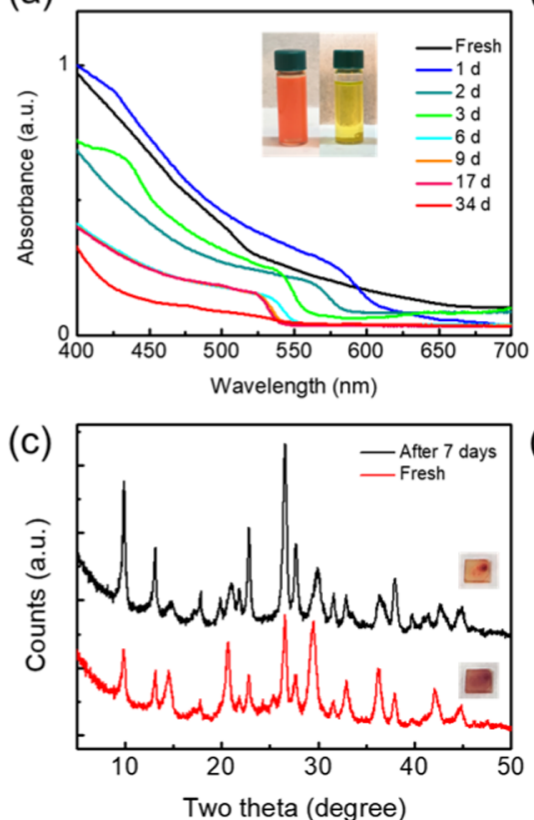

(b)
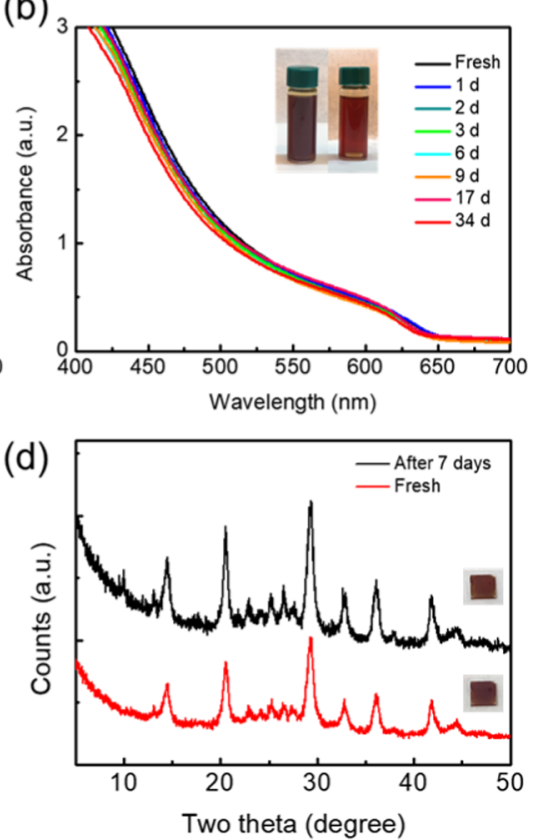

Figure 5. (a, b) UV-vis absorption spectra of the $\mathrm{CsPbI}_{2} \mathrm{Br} \mathrm{NCs}$ in a solution states as a function of storage time over 34 days in ambient conditions. (c, d) XRD profiles of the $\mathrm{CsPb}_{2} \mathrm{Br} \mathrm{NC}$ films in a fresh state and 7 day aged state.

(a)

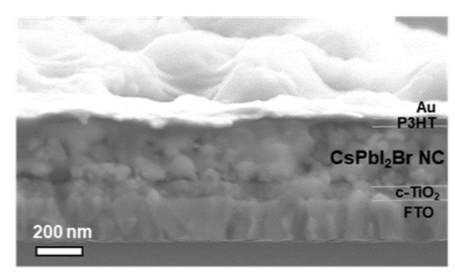

(c)

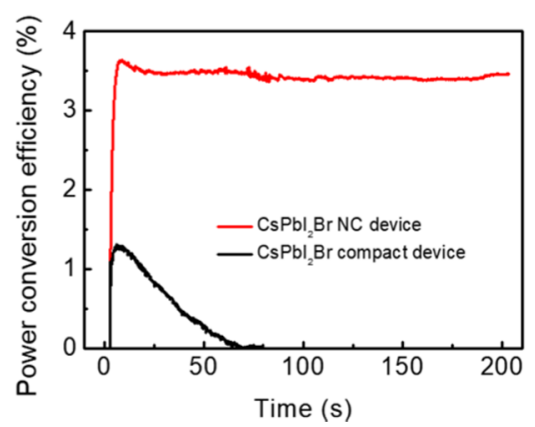

(b)

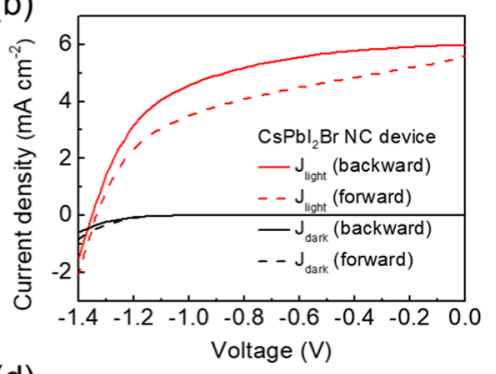

(d)

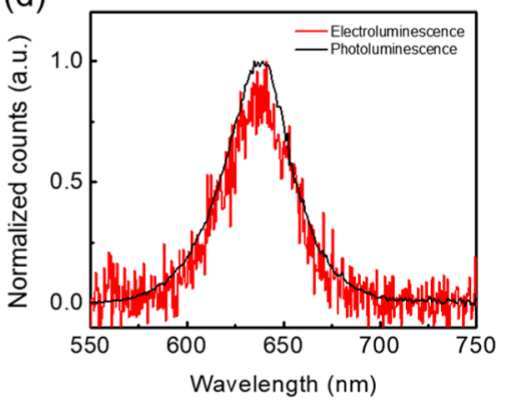

Figure 6. (a) Cross-sectional image of the $\mathrm{CsPbI}_{2} \mathrm{Br} \mathrm{NC}$ photovoltaic device. (b) $J-V$ curve of the $\mathrm{CB} / t-\mathrm{BuOH}$ CsPbI $\mathrm{Br} \mathrm{NC}_{\mathrm{photovoltaic}}$ device measured under 1 sun illumination in ambient conditions without encapsulations (red lines: $J_{\text {light }}$ black lines: $J_{\text {dark }}$ ). The photovoltaic parameters are the open-circuit voltage of $1.35 \mathrm{~V}$, short-circuit current density of $6.01 \mathrm{~mA} / \mathrm{cm}^{2}$, fill factor of 0.56 , and efficiency of $4.54 \%$ for a backward scan. (c) Maximum power point tracking for $200 \mathrm{~s}$ of the NC PV devices. (d) PL and EL spectra of the $\mathrm{CB} / t-\mathrm{BuOH} \mathrm{CsPbI}_{2} \mathrm{Br} \mathrm{NC}$ device under a forward bias.

evidence that the thin film retains the stable $\gamma$-phase perovskite also under charge injection.

\section{CONCLUSIONS}

We developed a novel method for the synthesis of $\mathrm{CsPb}_{2} \mathrm{Br}$ perovskite nanocrystals using an antisolvent mixture of $\mathrm{CB}$ and $t$-BuOH. It can be scaled up at room temperature in ambient environment. We demonstrate that $t-\mathrm{BuOH}$ acts as a crystallizing template for a successful nanocrystal formation, having an interaction with $\mathrm{Pb}^{2+}$ and passivating defect sites. With the passivation of $t-\mathrm{BuOH}$, the $\mathrm{CsPbI}_{2} \mathrm{Br} \mathrm{NC}$ shows improved stability of the black $\gamma$-perovskite phase during ambient storage of over 1 month. Finally, the photovoltaic device, incorporating NCs with a bandgap of $1.88 \mathrm{eV}$, showed a high open-circuit voltage $(1.35 \mathrm{~V})$ and enhanced stability compared to that of the compact $\mathrm{CsPb}_{2} \mathrm{Br}$ devices. We envision that this method is applicable for other types of other perovskite nanocrystals like $\mathrm{AMX}_{3}\left(\mathrm{~A}=\mathrm{Rb}^{+}, \mathrm{Cs}^{+} ; \mathrm{M}=\mathrm{Ge}^{2+}\right.$, 
$\mathrm{Sn}^{2+}, \mathrm{Ag}^{+}, \mathrm{Bi}^{3+} ; \mathrm{X}=\mathrm{F}^{-}, \mathrm{Cl}^{-}, \mathrm{Br}^{-}, \mathrm{I}^{-}$, or combinations thereof) in various optoelectronic applications, such as light-emitting diodes, photovoltaics, solar concentrators, and photon detection.

\section{EXPERIMENTAL SECTION}

Synthesis of $\mathrm{CsPbl}_{2} \mathrm{Br}$ QDs. All of the reagents were purchased from Sigma-Aldrich and used directly without further purification. In a synthesis of $\mathrm{CsPbI}_{2} \mathrm{Br}, \mathrm{PbI}_{2}(0.1 \mathrm{mmol}, \mathrm{TCI})$, and $\mathrm{CsBr}(0.1 \mathrm{mmol})$ were dissolved in $N, N$-dimethylformamide (DMF) $(1 \mathrm{~mL})$. Oleic acid $(\mathrm{OA}, 0.1 \mathrm{~mL})$ and oleylamine $(\mathrm{OAm}, 0.05 \mathrm{~mL})$, were added to stabilize the precursor solution. Then, $1 \mathrm{~mL}$ of the precursor solution was quickly added into the solvent mixture of chlorobenzene and $t$ butyl alcohol (in $10 \mathrm{~mL}$, ratio, 9:1) under vigorous stirring. Strong red emission was observed immediately after the injection. Then, methyl acetate is added into the NC solution to wash the NCs via precipitation. The obtained NC solution was further purified through centrifugation for $5 \mathrm{~min}$ at $10000 \mathrm{rpm}$, followed by redispersion of NCs in toluene (concentration $=60 \mathrm{mg} / \mathrm{mL}$ for device fabrication). Other samples with different colors were fabricated with a mixture of $\mathrm{PbX}_{2}$ and $\mathrm{CsX}(\mathrm{X}=\mathrm{Cl}, \mathrm{Br}, \mathrm{I})$. All of the above operations were implemented at room temperature under the fume hood.

Photovoltaic Device Fabrication. A fluorine-doped tin oxide (FTO)-coated glass was washed sequentially with detergent, deionized water, acetone, and isopropanol with ultrasonication for 10 min each. This was then dried with $\mathrm{N}_{2}$ and treated with $\mathrm{O}_{2}$ plasma. The clean substrate was coated with a $\mathrm{TiCl}_{4}$ aqueous solution, followed by annealing at $500{ }^{\circ} \mathrm{C}$ for $30 \mathrm{~min}$ in air to form a compact n-type blocking layer of $\mathrm{TiO}_{2}$. The $\mathrm{CsPb}_{2} \mathrm{Br} \mathrm{NC}$ films were fabricated using repetitive spin coating and washing steps. For the ligand washing procedure, saturated $\mathrm{Pb}\left(\mathrm{NO}_{3}\right)_{2}$ in $\mathrm{MeOAc}$ was prepared by sonicating $40 \mathrm{mg}$ of $\mathrm{Pb}\left(\mathrm{NO}_{3}\right)_{2}$ in $40 \mathrm{~mL}$ of $\mathrm{MeOAc}$ for $10 \mathrm{~min}$ and then centrifuged at $4000 \mathrm{rpm}$ for $5 \mathrm{~min}$ to remove the excess salt. Each layer of $\mathrm{CsPbI}_{2} \mathrm{Br}$ QDs was spin-coated from the NC solution in toluene $(60 \mathrm{mg} / \mathrm{mL})$ at spin speeds of $800 \mathrm{rpm}$ for $20 \mathrm{~s}$ and $2000 \mathrm{rpm}$ for $20 \mathrm{~s}$. Then, the film was dipped into $\mathrm{Pb}\left(\mathrm{NO}_{3}\right)_{2}$ in $\mathrm{MeOAc}$ solution, rinsed in neat $\mathrm{MeOAc}$, and then immediately dried with nitrogen. A thickness of $\sim 300 \mathrm{~nm}$ was achieved by repeating the above-mentioned steps four or five times. The hole-transporting material, poly(3-hexylthiophene) was spin-coated at $2000 \mathrm{rpm}$ for 30 $\mathrm{s}$ from a solution of $10 \mathrm{mg}$ of P3HT in $1 \mathrm{~mL}$ of chlorobenzene. Au was evaporated at a rate ranging from 0.5 to $1 \AA / \mathrm{s}$ for a total thickness of $70 \mathrm{~nm}$.

Characterizations. UV-vis absorption spectra are obtained with UV-vis Varian Cary 5000. The BF-TEM images and selected area electron diffraction (SAED) patterns were obtained by means of a JEM-1400Plus and JEM-ARM200F instrument, equipped with a thermionic source (LaB6), and operated at $120 \mathrm{kV}$. Data were acquired with a Gatan Orius 830 CCD camera $(2048 \times 2048$ active pixels). For TEM analyses, the nanocrystals were placed on carboncoated TEM Cu grids in a glovebox via drop casting. The grids were kept under an inert atmosphere for a few minutes before the TEM analyses. The perovskite NC films were prepared on bare glass substrates. XRD patterns were recorded with a Bruker D8 Advance diffractometer with a Bragg-Brentano geometry equipped with a $\mathrm{Cu}$ $\mathrm{K} \alpha_{1}(\lambda=1.544060 \AA)$ anode, operating at $40 \mathrm{kV}$ and $40 \mathrm{~mA}$. All of the diffraction patterns were collected at room temperature, with a grazing incidence mode at an incidence of $1.5^{\circ}$. The FT-IR spectra of the perovskite films deposited on silicon substrates were measured using a PerkinElmer 2000 FT-IR spectrometer in the transmission mode. Measurements were carried out in the $100-4000 \mathrm{~cm}^{-1}$ range. The substrate contribution is removed from the measured data. Scanning electron microscopy (SEM) images were obtained using JCM-6010LV, JEOL at $15-20 \mathrm{kV}$ electron beam. The current density-voltage $(J-V)$ characteristics were measured with a computer-controlled Keithley 2420 source meter in air without any device encapsulation. The simulated Air Mass 1.5 Global (AM 1.5G) irradiance was provided with a class AAA Newport solar simulator. For the $J-V$ measurement, the scan rates were $0.1 \mathrm{~V} / \mathrm{s}$.

\section{ASSOCIATED CONTENT}

\section{Supporting Information}

The Supporting Information is available free of charge at https://pubs.acs.org/doi/10.1021/acs.chemmater.0c03463.

Experimental details for UV-vis absorbance, PL, crystal structure, NMR spectra, time-resolved PL, FT-IR, statistics of device parameters, and degradation test of NCs (PDF)

\section{AUTHOR INFORMATION}

\section{Corresponding Authors}

Min Kim - Center for Nano Science and Technology@PoliMi, Istituto Italiano di Tecnologia, 20133 Milan, Italy; School of Semiconductor and Chemical Engineering, Jeonbuk National University, Jeonju 54896, Republic of Korea;

Email: minkim@jbnu.ac.kr

Annamaria Petrozza - Center for Nano Science and Technology@PoliMi, Istituto Italiano di Tecnologia, 20133 Milan, Italy; ๑ orcid.org/0000-0001-6914-4537;

Email: annamaria.petrozza@iit.it

\section{Authors}

Daniele Cortecchia - Center for Nano Science and Technology@PoliMi, Istituto Italiano di Tecnologia, 20133 Milan, Italy; ๑ orcid.org/0000-0001-8623-9191

Tetiana Borzda - Center for Nano Science and Technology @ PoliMi, Istituto Italiano di Tecnologia, 20133 Milan, Italy

Wojciech Mróz - Center for Nano Science and Technology@ PoliMi, Istituto Italiano di Tecnologia, 20133 Milan, Italy

Luca Leoncino - Electron Microscopy Facility, Istituto Italiano di Tecnologia, Genova 16163, Italy

David Dellasega - Department of Energy, Politecnico di Milano, 20133 Milan, Italy

Soo-Hyoung Lee - School of Semiconductor and Chemical Engineering, Jeonbuk National University, Jeonju 54896, Republic of Korea; $\odot$ orcid.org/0000-0002-2730-5723

Complete contact information is available at: https://pubs.acs.org/10.1021/acs.chemmater.0c03463

\section{Notes}

The authors declare no competing financial interest.

\section{ACKNOWLEDGMENTS}

M.K. acknowledges funding from EU Horizon 2020 via a Marie Sklodowska Curie Fellowship (Project No. 797546, FASTEST). This research was also supported by the National Research Foundation of Korea (NRF) grant funded by the Korean government (MSIT) (NRF-2020R1F1A1073482). The authors thank the Center for University-wide Research Facilities (CURF) at Jeonbuk National University for TEM analysis.

\section{REFERENCES}

(1) Akkerman, Q. A.; D’Innocenzo, V.; Accornero, S.; Scarpellini, A.; Petrozza, A.; Prato, M.; Manna, L. Tuning the Optical Properties of Cesium Lead Halide Perovskite Nanocrystals by Anion Exchange Reactions. J. Am. Chem. Soc. 2015, 137, 10276-10281.

(2) Nedelcu, G.; Protesescu, L.; Yakunin, S.; Bodnarchuk, M. I.; Grotevent, M. J.; Kovalenko, M. V. Fast Anion-Exchange in Highly Luminescent Nanocrystals of Cesium Lead Halide Perovskites (CsPbX3, X = Cl, Br, I). Nano Lett. 2015, 15, 5635-5640.

(3) Bekenstein, Y.; Koscher, B. A.; Eaton, S. W.; Yang, P.; Alivisatos, A. P. Highly Luminescent Colloidal Nanoplates of Perovskite Cesium 
Lead Halide and Their Oriented Assemblies. J. Am. Chem. Soc. 2015, 137, 16008-16011.

(4) Song, J.; Li, J.; Li, X.; Xu, L.; Dong, Y.; Zeng, H. Quantum Dot Light-Emitting Diodes Based on Inorganic Perovskite Cesium Lead Halides (CsPbX3). Adv. Mater. 2015, 27, 7162-7167.

(5) Chiba, T.; Hayashi, Y.; Ebe, H.; Hoshi, K.; Sato, J.; Sato, S.; Pu, Y.-J.; Ohisa, S.; Kido, J. Anion-exchange red perovskite quantum dots with ammonium iodine salts for highly efficient light-emitting devices. Nat. Photonics 2018, 12, 681-687.

(6) Swarnkar, A.; Marshall, A. R.; Sanehira, E. M.; Chernomordik, B. D.; Moore, D. T.; Christians, J. A.; Chakrabarti, T.; Luther, J. M. Quantum dot-induced phase stabilization of $\alpha$-CsPbI3 perovskite for high-efficiency photovoltaics. Science 2016, 354, 92-95.

(7) Akkerman, Q. A.; Gandini, M.; Di Stasio, F.; Rastogi, P.; Palazon, F.; Bertoni, G.; Ball, J. M.; Prato, M.; Petrozza, A.; Manna, L. Strongly emissive perovskite nanocrystal inks for high-voltage solar cells. Nat. Energy 2017, 2, No. 16194.

(8) Yakunin, S.; Protesescu, L.; Krieg, F.; Bodnarchuk, M. I.; Nedelcu, G.; Humer, M.; De Luca, G.; Fiebig, M.; Heiss, W.; Kovalenko, M. V. Low-threshold amplified spontaneous emission and lasing from colloidal nanocrystals of caesium lead halide perovskites. Nat. Commun. 2015, 6, No. 8056.

(9) Grätzel, M. The light and shade of perovskite solar cells. Nat. Mater. 2014, 13, 838.

(10) Sutton, R. J.; Eperon, G. E.; Miranda, L.; Parrott, E. S.; Kamino, B. A.; Patel, J. B.; Hörantner, M. T.; Johnston, M. B.; Haghighirad, A. A.; Moore, D. T.; Snaith, H. J. Bandgap-Tunable Cesium Lead Halide Perovskites with High Thermal Stability for Efficient Solar Cells. Adv. Energy Mater. 2016, 6, No. 1502458.

(11) Sharma, S.; Weiden, N.; Weiss, A. Phase Diagrams of Quasibinary Systems of the Type: ABX3 - A'BX3; ABX3 $\mathrm{AB}^{\prime} \mathrm{X} 3$, and $\mathrm{ABX} 3-\mathrm{ABX}^{\prime} 3 ; \mathrm{X}=$ Halogen. Z. Phys. Chem. 1992, 175, 63-80.

(12) Yuan, G.; Ritchie, C.; Ritter, M.; Murphy, S.; Gómez, D. E.; Mulvaney, P. The Degradation and Blinking of Single CsPbI3 Perovskite Quantum Dots. J. Phys. Chem. C 2018, 122, 13407-13415. (13) An, R.; Zhang, F.; Zou, X.; Tang, Y.; Liang, M.; Oshchapovskyy, I.; Liu, Y.; Honarfar, A.; Zhong, Y.; Li, C.; Geng, H.; Chen, J.; Canton, S. E.; Pullerits, T.; Zheng, K. Photostability and Photodegradation Processes in Colloidal CsPbI3 Perovskite Quantum Dots. ACS Appl. Mater. Interfaces 2018, 10, 39222-39227.

(14) Protesescu, L.; Yakunin, S.; Bodnarchuk, M. I.; Krieg, F.; Caputo, R.; Hendon, C. H.; Yang, R. X.; Walsh, A.; Kovalenko, M. V. Nanocrystals of Cesium Lead Halide Perovskites (CsPbX3, X $=\mathrm{Cl}$, $\mathrm{Br}$, and I): Novel Optoelectronic Materials Showing Bright Emission with Wide Color Gamut. Nano Lett. 2015, 15, 3692-3696.

(15) Dutta, A.; Dutta, S. K.; Das Adhikari, S.; Pradhan, N. PhaseStable CsPbI3 Nanocrystals: The Reaction Temperature Matters. Angew. Chem., Int. Ed. 2018, 57, 9083-9087.

(16) Pu, Y.; Cai, F.; Wang, D.; Wang, J.-X.; Chen, J.-F. Colloidal Synthesis of Semiconductor Quantum Dots toward Large-Scale Production: A Review. Ind. Eng. Chem. Res. 2018, 57, 1790-1802.

(17) Hu, M. Z.; Zhu, T. Semiconductor Nanocrystal Quantum Dot Synthesis Approaches Towards Large-Scale Industrial Production for Energy Applications. Nanoscale Res. Lett. 2015, 10, No. 469.

(18) Sun, S.; Yuan, D.; Xu, Y.; Wang, A.; Deng, Z. Ligand-Mediated Synthesis of Shape-Controlled Cesium Lead Halide Perovskite Nanocrystals via Reprecipitation Process at Room Temperature. ACS Nano 2016, 10, 3648-3657.

(19) Li, X.; Wu, Y.; Zhang, S.; Cai, B.; Gu, Y.; Song, J.; Zeng, H. CsPbX3 Quantum Dots for Lighting and Displays: Room-Temperature Synthesis, Photoluminescence Superiorities, Underlying Origins and White Light-Emitting Diodes. Adv. Funct. Mater. 2016, 26, 24352445.

(20) Levchuk, I.; Osvet, A.; Tang, X.; Brandl, M.; Perea, J. D.; Hoegl, F.; Matt, G. J.; Hock, R.; Batentschuk, M.; Brabec, C. J. Brightly Luminescent and Color-Tunable Formamidinium Lead Halide Perovskite FAPbX3 (X = Cl, Br, I) Colloidal Nanocrystals. Nano Lett. 2017, 17, 2765-2770.
(21) Zhang, X.; Bai, X.; Wu, H.; Zhang, X.; Sun, C.; Zhang, Y.; Zhang, W.; Zheng, W.; Yu, W. W.; Rogach, A. L. Water-Assisted Size and Shape Control of CsPbBr3 Perovskite Nanocrystals. Angew. Chem., Int. Ed. 2018, 57, 3337-3342.

(22) Ghosh, D.; Ali, M. Y.; Chaudhary, D. K.; Bhattacharyya, S. Dependence of halide composition on the stability of highly efficient all-inorganic cesium lead halide perovskite quantum dot solar cells. Sol. Energy Mater. Sol. Cells 2018, 185, 28-35.

(23) Christodoulou, S.; Di Stasio, F.; Pradhan, S.; Stavrinadis, A.; Konstantatos, G. High-Open-Circuit-Voltage Solar Cells Based on Bright Mixed-Halide CsPbBrI2 Perovskite Nanocrystals Synthesized under Ambient Air Conditions. J. Phys. Chem. C 2018, 122, 76217626.

(24) Munjal, B.; Bansal, A. K. Impact of Tert-Butyl Alcohol on Crystallization Kinetics of Gemcitabine Hydrochloride in Frozen Aqueous Solutions. J. Pharm. Sci. 2015, 104, 87-97.

(25) Woo Choi, J.; Woo, H. C.; Huang, X.; Jung, W.-G.; Kim, B.-J.; Jeon, S.-W.; Yim, S.-Y.; Lee, J.-S.; Lee, C.-L. Organic-inorganic hybrid perovskite quantum dots with high PLQY and enhanced carrier mobility through crystallinity control by solvent engineering and solid-state ligand exchange. Nanoscale 2018, 10, 13356-13367.

(26) Zhao, B.; Jin, S.-F.; Huang, S.; Liu, N.; Ma, J.-Y.; Xue, D.-J.; Han, Q.; Ding, J.; Ge, Q.-Q.; Feng, Y.; Hu, J.-S. Thermodynamically Stable Orthorhombic $\gamma$-CsPbI3 Thin Films for High-Performance Photovoltaics. J. Am. Chem. Soc. 2018, 140, 11716-11725.

(27) Lomas, J. S. 1H NMR spectra of alcohols in hydrogen bonding solvents: DFT/GIAO calculations of chemical shifts. Magn. Reson. Chem. 2016, 54, 28-38.

(28) Hamill, J. C.; Schwartz, J.; Loo, Y.-L. Influence of Solvent Coordination on Hybrid Organic-Inorganic Perovskite Formation. ACS Energy Lett. 2018, 3, 92-97.

(29) Zhang, H.; Fu, X.; Tang, Y.; Wang, H.; Zhang, C.; Yu, W. W.; Wang, X.; Zhang, Y.; Xiao, M. Phase segregation due to ion migration in all-inorganic mixed-halide perovskite nanocrystals. Nat. Commun. 2019, 10, No. 1088.

(30) Zeng, Q.; Zhang, X.; Feng, X.; Lu, S.; Chen, Z.; Yong, X.; Redfern, S. A. T.; Wei, H.; Wang, H.; Shen, H.; Zhang, W.; Zheng, W.; Zhang, H.; Tse, J. S.; Yang, B. Polymer-Passivated Inorganic Cesium Lead Mixed-Halide Perovskites for Stable and Efficient Solar Cells with High Open-Circuit Voltage over 1.3 V. Adv. Mater. 2018, 30, No. 1705393.

(31) Liu, C.; Zeng, Q.; Yang, B. Managing Energy Loss in Inorganic Lead Halide Perovskites Solar Cells. Adv. Mater. Interfaces 2019, 6, No. 1901136

(32) Li, N.; Zhu, Z.; Li, J.; Jen, A. K.-Y.; Wang, L. Inorganic CsPb1$\mathrm{xSnxIBr2}$ for Efficient Wide-Bandgap Perovskite Solar Cells. Adv. Energy Mater. 2018, 8, No. 1800525.

(33) Guo, Z.; Zhao, S.; Liu, A.; Kamata, Y.; Teo, S.; Yang, S.; Xu, Z.; Hayase, S.; Ma, T. Niobium Incorporation into CsPbI2Br for Stable and Efficient All-Inorganic Perovskite Solar Cells. ACS Appl. Mater. Interfaces 2019, 11, 19994-20003.

(34) Zhou, L.; Guo, X.; Lin, Z.; Ma, J.; Su, J.; Hu, Z.; Zhang, C.; Liu, S.; Chang, J.; Hao, Y. Interface engineering of low temperature processed all-inorganic $\mathrm{CsPbI} 2 \mathrm{Br}$ perovskite solar cells toward PCE exceeding 14\%. Nano Energy 2019, 60, 583-590.

(35) Hazarika, A.; Zhao, Q.; Gaulding, E. A.; Christians, J. A.; Dou, B.; Marshall, A. R.; Moot, T.; Berry, J. J.; Johnson, J. C.; Luther, J. M. Perovskite Quantum Dot Photovoltaic Materials beyond the Reach of Thin Films: Full-Range Tuning of A-Site Cation Composition. ACS Nano 2018, 12, 10327-10337. 